\title{
Analysis of one-dimensional advection-diffusion model with variable coefficients describing solute transport in a porous medium
}

\author{
Munshoor Ahmed, Qurat Ul Ain Zainab, \\ Shamsul Qamar
}

Received: date / Accepted: date

\begin{abstract}
This work presents the analytical solution and temporal moments of one-dimensional advection-diffusion model with variable coefficients. Two case studies along with the two different sets of boundary conditions are considered at the inlet and outlet of the domain. In the first case, a time dependent solute dispersion in the homogeneous domain along uniform flow is taken into account, whereas in the second case, due to inhomogeneity of domain, velocity is taken spatially dependent and the dispersion is assumed proportional to the square of the velocity. The Laplace transform is used to obtain the analytical solutions. The analytical temporal moments are derived from the Laplace domain solutions. To verify the correctness of the analytical solutions, a high resolution second order finite volume scheme is applied. Different case studies are considered and discussed. Both analytical and numerical results are in good agreement with each other.
\end{abstract}

Keywords Advection-diffusion equation · rectangular pulse injections · analytical solutions · moment analysis · finite volume method · dynamic simulations.

Mathematics Subject Classification (2000) 80A23 $\cdot 26 \mathrm{~A} 33 \cdot 42 \mathrm{~A} 16$

Munshoor Ahmed (Corresponding Author)

Department of Mathematics, COMSATS Institute of Information Technology, Islamabad, Pakistan.

Tel.: +9251 8318471

Fax: +92-51-8318499

E-mail: manshoor@comsats.edu.pk

Quratul Ain Zanib

Department of Mathematics, COMSATS Institute of Information Technology, Islamabad, Pakistan.

E-mail: quratulain.zainab@yahoo.com

Shamsul Qamar

Department of Mathematics, COMSATS Institute of Information Technology, Islamabad, Pakistan

Max Planck Institute for Dynamics of Complex Technical Systems,Sandtorstrasse 1, 39106 Magdeburg, Germany

E-mail: shamsul.qamar@comsats.edu.pk 


\section{Nomenclature}

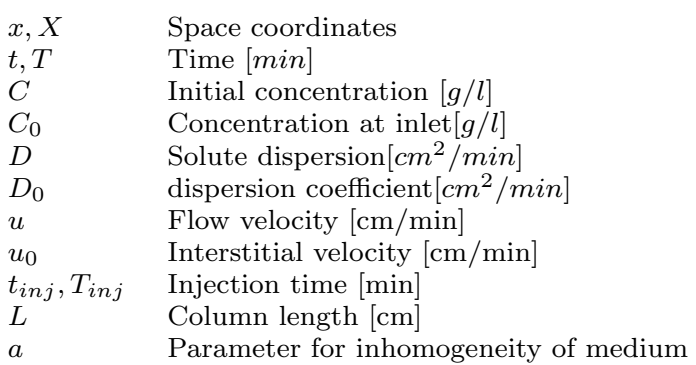

\section{Introduction}

Advection-diffusion equations (ADEs) are widely used for the simulation of different processes in the areas of environmental sciences, bio sciences, chemical engineering and hydrology. In nature, the fluid transport takes place due to combined effect of convection and diffusion. An ADE is a parabolic type PDE based on conservation laws. Its analytical and numerical solutions are useful for understanding the pollutant or contaminant concentration distribution behavior through an open medium like rivers, lake, air and porous medium like aquifer. Moreover, these solutions have applications in areas of environmental sciences, bio sciences, chemical engineering and hydrology. For example, Lanser and Jan [1] analyzed the operator splitting for advection-diffusion-reaction problems from air pollution. In [2] Samadi et. al discussed the effect of the physical properties of the substrate on the kinetics of cell adhesion and crawling studied by an axisymmetric diffusion-energy balance coupled model. Grisak and Pickens in [3] give analytical solution for solute transport through fractured media with matrix diffusion. Applications of the spur diffusion model to the radiation chemistry of aqueous solutions are presented in [4]. Furthermore, these equations are also used to model chromatographic processes and ground flows [5].

Several approaches have been applied for solving the linear ADEs analytically $[6-12,14]$. As an example, the first approach was to reduce the ADE into diffusion equation by hiding the convective term(s). This task was accomplished by introducing moving coordinates [6-10] or by proposing another dependent variable $[13,14]$. Several techniques, like, Hankel transform method, Aris moment method, perturbation method, superposition method have been used to solve ADEs. In [15] Thongmoon and McKibbin, discussed numerical solutions obtained from cubic splines, finite difference schemes and Crank-Nicolson methods and compared these results with analytical solution. However, in the present article, the Laplace transformation(LT) technique [24] is applied to solve the model equation and to derive analytical moments using the moment generating property of Laplace domain solutions. Moreover, in the cases where the inverse Laplace transform is not possible, numerical inverse Laplace is applied, see [16-23].

In this manuscript, we have obtained analytical solutions of ADE for two problems. In the first problem, time dependent dispersion coefficient in homogeneous 
domain is considered. While, in the second problem the solution is found for a space dependent advection coefficient in nonhomogeneous domain and dispersion coefficient is assumed proportional to the square of the velocity, as considered in [25]. The present work is an extension of $[25,26]$ in which the authors have used the continuous Dirichlet type boundary conditions only.

In this manuscript we have derived analytical solutions and temporal moments for both uniform and non-uniform flows considering two different sets of boundary conditions which cover both continuous and pulse injections. According to our knowledge, no one has studied these problems to such a depth. Beyond deriving analytical solutions for two sets of BCs, we have derived moments up to third order. In the literature, the researchers have restricted themselves up to simple continuous Dirichlet BCs and moments derivation up to second order. Thus, our solutions are more general and we went few steps beyond all previous studies [2529]. Moreover, it is important to mention that the Danckwerts type boundary conditions are more appropriate for the solution of current model equations which account for back mixing in the case of large axial dispersion [30].

For further analysis, the first three statistical temporal moments are determined from solutions in the Laplace domain. These moments are used to understand the effect of different kinetic parameters appearing in the model equations. Furthermore, the derived analytical solution could be used to analyze the accuracy of new numerical schemes. Extensive discussion on moment analysis is done in literature $[31,32]$. In these partly classical papers analytical expressions have been generated for specific solute transport in porous medium and boundary conditions (BCs). This analysis typically covers just the most important first and second moments, i.e. retention times and band broadening. Since the influence of the boundary conditions is often not discussed in sufficient detail, we have further compared the moment expressions for Danckwerts and Dirichlet BCs. To validate the analytical solutions and the moment expressions, a high resolution finite volume scheme (HR-FVS) of Koren [33,34] is employed. Moreover, different parametric case studies are considered.

The derived solutions and moments could be used to optimize experimental conditions, to improve the product quality and to validate new numerical schemes derived for solving these problems. Moreover, the derived moments can be used to estimate model parameters from the experimental moments [30-32].

\section{Mathematical Equation}

In this section one-dimensional advection-diffusion equation with variable coefficients is presented [30]. The mathematical form of this equation is:

$$
\frac{\partial C}{\partial t}=\frac{\partial}{\partial x}\left(D(x, t) \frac{\partial C}{\partial x}-u(x, t) C\right)
$$

where $C$ is the solute concentration at position $x$ at time $t$. Moreover, $D$ is the solute dispersion, it is known as dispersion coefficient if it is independent of time and position and $u$ is the medium's flow velocity. It can be noted that suitable boundary conditions are needed to solve Eq. (1). The domain length is taken as $x=L$ and two sets of boundary conditions along with an initial condition are given below. 
The initial concentration is taken as

$$
C(x, 0)=0 .
$$

One of the following boundary conditions can be used to close the problem.

\section{Type I boundary condition:}

The Dirichlet inlet boundary condition with pulse injection $t_{i n j}$

$$
C(0, t)=\left\{\begin{array}{c}
C_{0}, \quad 0<t \leq t_{i n j}, \\
0, \quad t>t_{i n j}
\end{array}\right.
$$

Moreover, at the outlet the following Neuman boundary condition can be applied.

$$
\left.\frac{\partial C(x, t)}{\partial x}\right|_{x=\infty}=0, \quad t \geq 0 .
$$

Here, $t_{i n j}$ is the time of injection of the pulse and $C_{0}$ is the concentration of the injected pulse. The right boundary for Dirichlet (first type) BCs is at infinity. In this case, we assume that the channel (column) length is hypothetically infinite. This assumption for the considered particular boundary conditions is required (necessary) to satisfy the overall mass balance. In other words, the mass injected at the inlet of the column (channel) should be equal to the mass at the outlet, called as mass balance.

Type II boundary condition: The Danckwerts boundary condition

$$
-\frac{D(x, t)}{u(x, t)} \frac{\partial C}{\partial x}+\left.C\right|_{x=0}=\left\{\begin{array}{lr}
C_{0}, & \text { if } 0<t \leq t_{i n j}, \\
0, & t>t_{i n j},
\end{array}\right.
$$

and the following Neumann outflow boundary condition is used:

$$
\left.\frac{\partial C(x, t)}{\partial x}\right|_{x=L}=0, \quad t \geq 0
$$

While, in the case of Danckwerts BC, which is more general, the consideration of finite length column satisfy the required mass balance.

In the following we consider two case studies, namely, uniform flow for the time dependent dispersion and non-uniform flow for the space dependent dispersion coefficient.

2.1 Uniform flow for time dependent dispersion coefficient

In this case, initially solute free domain, i.e. $u(x, 0)=0$ and a uniform input concentration, are considered. So we take,

$$
D(x, t)=D_{0} f(m t), \quad u(x, t)=u_{0},
$$

where $m$ is the coefficient having dimension inverse to that of time variable. Therefore, $f(m t)$ is a dimensionless expression in variable $m t$. However, we take $f(m t)=1$ for $m=0$ or $t=0$, see [25]. The coefficients $D_{0}$ and $u_{0}$ are dispersion coefficient and uniform velocity, respectively. Using Eq. (7) into Eq. (1) we get 


$$
\frac{\partial C}{\partial t}=D_{0} f(m t) \frac{\partial^{2} C}{\partial x^{2}}-u_{0} \frac{\partial C}{\partial x} .
$$

Here, new time and space independent variables are introduced by defining the following transformations so that we can use Laplace transformation conveniently [25]:

$$
X=\frac{x}{f(m t)} \quad \text { or } \quad \frac{d X}{d x}=\frac{1}{f(m t)} .
$$

It can be noted that $X$ has the same dimension as $x$ because $m t$ is a nondimensional term. Moreover, for the time dependent coefficient the following transformation is used [25]:

$$
T=\frac{t}{f(m t)} \quad \text { or } \quad \frac{d T}{d t}=\frac{1}{f(m t)}
$$

where dimension of $T$ is same as $t$. The nature of initial condition in new time domain depends upon the choice of $f(m t)$ so it should be chosen in a such a manner which do not change the nature of initial conditions. By using these transformations, the Eq. (8) along with initial condition given by Eq. (2) in transformed form become:

$$
\begin{gathered}
\frac{\partial^{2} C}{\partial X^{2}}-\frac{u_{0}}{D_{0}} \frac{\partial C}{\partial X}-\frac{1}{D_{0}} \frac{\partial C}{\partial T}=0, \\
C(X, 0)=0, \quad 0 \leq X \leq X_{0}, \quad T=0 ; \quad X_{0}=\frac{L}{f(m t)} .
\end{gathered}
$$

In the same manner, the boundary conditions given in Eqs. (3)-(6) are transformed as under.

The Dirichlet boundary condition:

$$
C(X, T)=\left\{\begin{array}{c}
C_{0}, \quad 0<T \leq T_{i n j}, \\
0, \quad T>T_{i n j} .
\end{array}\right.
$$

Moreover, the outlet Neumann boundary condition is given as

$$
\frac{\partial C(X, T)}{\partial X}=0, \quad X=\infty, \quad T \geq 0
$$

The transformed Danckwerts boundary condition is

$$
-D(X, T) \frac{\partial C}{\partial X}+\left.u(X, T) C\right|_{X=0}=\left\{\begin{array}{lc}
u_{0} C_{0}, & \text { if } 0<T \leq T_{i n j}, \\
0, & T>T_{i n j},
\end{array}\right.
$$

and Neumann outflow boundary condition is

$$
\frac{\partial C(X, T)}{\partial X}=0, \quad X=X_{0}, \quad T \geq 0 .
$$


The Laplace transformation is defined as

$$
\bar{c}(s, X)=\int_{0}^{\infty} e^{-s T} c(T, X) d T, \quad T \geq 0 .
$$

Applying the Laplace transformation on Eq. (11), we get

$$
\frac{\partial^{2} \bar{C}}{\partial X^{2}}-\frac{u_{0}}{D_{0}} \frac{\partial \bar{C}}{\partial X}-\frac{1}{D_{0}} s \bar{C}=0
$$

The solution of this equation is given as

$$
\bar{C}(X, s)=A e^{m_{1} X}+B e^{m_{2} X},
$$

where

$$
m_{1,2}=\frac{u_{0}}{2 D_{0}} \pm \sqrt{\frac{u_{0}^{2}}{4 D_{0}^{2}}+\frac{s}{D_{0}}} .
$$

The integration constants $A$ and $B$ in Eq. (19) can be obtained by utilizing type I and type II boundary conditions given in Eqs. (13) and (14). However, first we transform these conditions in the Laplace domain as under

$$
\begin{gathered}
\bar{C}(X, s)=\frac{C_{0}\left(1-e^{-s T_{i n j}}\right)}{s} \quad, X=0, \\
\frac{\partial \bar{C}(X, s)}{\partial X}=0, \quad X=\infty, \quad T \geq 0 .
\end{gathered}
$$

Using Eqs. (21) and (22) we get the values of $A$ and $B$ as follow

$$
A=0, \quad B=\frac{C_{0}\left(1-e^{-s T_{i n j}}\right)}{s} \quad, X=0 .
$$

Using $A$ and $B$ in Eq. (19), we get solution as

$$
\bar{C}(X, s)=\frac{C_{0}\left(1-e^{-s T_{i n j}}\right)}{s} e^{m_{2} X} .
$$

Analytical inverse transformation can be applied to obtain the solution in the actual time domain. Thus, the time domain solution $C(X, T)$ is obtained as

$$
C(X, T)=\frac{1}{2 \pi i} \int_{\gamma-i \infty}^{\gamma+i \infty} e^{-T s} C(X, s) d s,
$$

where, $\gamma$ is a real constant that exceeds the real part of all the singularities of $C(X, s)$. Thus, we obtain [35]:

$$
C(X, T)=\left\{\begin{array}{lr}
C_{0} M(X, T), & 0<T \leq T_{\mathrm{inj}} \\
C_{0} M(X, T)-C_{0} M\left(X, T-T_{\mathrm{inj}}\right), \quad T>T_{\mathrm{inj}}
\end{array}\right.
$$


where

$$
M(X, T)=\frac{1}{2} \operatorname{erfc}\left[\frac{\frac{X}{D_{0}}-\frac{u_{0} T}{D_{0}}}{2 \sqrt{\frac{T}{D_{0}}}}\right]+\frac{1}{2} e^{\frac{u_{0} X}{D_{0}}} \operatorname{erfc}\left[\frac{\frac{X}{D_{0}}+\frac{u_{0} T}{D_{0}}}{2 \sqrt{\frac{T}{D_{0}}}}\right],
$$

Here, erfc denotes the complementary error function.

We will now use the type II boundary conditions given by Eqs. (15) and (16). Before applying the conditions we transform them in the Laplace domain as under:

$$
\begin{gathered}
\bar{C}(X, s)=\frac{C_{0}\left(1-e^{-s T_{i n j}}\right)}{s}+\frac{D_{0}}{u_{0}} \frac{\partial \bar{C}}{\partial X}, \quad X=0, \\
\frac{\partial \bar{C}(X, s)}{\partial X}=0, \quad X=X_{0}, \quad T \geq 0 .
\end{gathered}
$$

On using Eqs. (28) and (29) in Eq. (19), we obtained A and B as

$$
\begin{gathered}
A=-\frac{C_{0}}{s}\left(1-e^{-s T_{i n j}}\right)\left[\frac{m_{2} e^{m_{2} X_{0}}}{m_{1} e^{m_{1} X_{0}}\left(1-\frac{m_{2} D_{0}}{u_{0}}\right)-m_{2} e^{m_{2} X_{0}}\left(1-\frac{m_{1} D_{0}}{u_{0}}\right)}\right], \\
B=\frac{C_{0}}{s}\left(1-e^{-s T_{i n j}}\right)\left[\frac{m_{1} e^{m_{1} X_{0}}}{m_{1} e^{m_{1} X_{0}}\left(1-\frac{m_{2} D_{0}}{u_{0}}\right)-m_{2} e^{m_{2} X_{0}}\left(1-\frac{m_{1} D_{0}}{u_{0}}\right)}\right] .
\end{gathered}
$$

On substituting these values of A and B in Eq. (19), we obtain

$$
\begin{gathered}
\bar{C}(X, s)=\frac{C_{0}\left(1-e^{-s T_{i n j}}\right)}{s\left(m_{1} e^{m_{1} X_{0}}\left(1-\frac{m_{2} D_{0}}{u_{0}}\right)-m_{2} e^{m_{2} X_{0}}\left(1-\frac{m_{1} D_{0}}{u_{0}}\right)\right)} \\
{\left[m_{1} e^{m_{1} X_{0}+m_{2} X}-m_{2} e^{m_{2} X_{0}+m_{1} X}\right] .}
\end{gathered}
$$

The analytical inverse Laplace transformation gives the following solution in the actual time domain [35]:

$$
C(X, T)=\left\{\begin{array}{lr}
C_{0} H_{1}(X, T), & 0<T \leq T_{\mathrm{inj}}, \\
C_{0} H_{1}(X, T)-C_{0} H_{1}\left(X, T-T_{\mathrm{inj}}\right), \quad T>T_{\mathrm{inj}}
\end{array}\right.
$$

where

$$
\begin{aligned}
H_{1}(T, X)= & \frac{1}{2} \operatorname{erfc}\left[\frac{\frac{X}{D_{0}}-\frac{u_{0} T}{D_{0}}}{2 \sqrt{\frac{T}{D_{0}}}}\right]+\left(\frac{\left(\frac{u_{0}}{D_{0}}\right)^{2} T}{\pi \frac{1}{D_{0}}}\right)^{\frac{1}{2}} \exp \left[-\frac{\left(\frac{X}{D_{0}}-\frac{u_{0} T}{D_{0}}\right)^{2}}{4 \frac{T}{D_{0}}}\right] \\
& -\frac{1}{2}\left(1+\frac{u_{0} X}{D_{0}}+\frac{\left(\frac{u_{0}}{D_{0}}\right)^{2} T}{\frac{1}{D_{0}}}\right) e^{\frac{u_{0} X}{D_{0}}} \operatorname{erfc}\left[\frac{\frac{X}{D_{0}}+\frac{u_{0} T}{D_{0}}}{2 \sqrt{\frac{T}{D_{0}}}}\right] \\
& +\left(\frac{4\left(\frac{u_{0}}{D_{0}}\right)^{2} T}{\pi \frac{1}{D_{0}}}\right)^{\frac{1}{2}}\left[1+\frac{\frac{u_{0}}{D_{0}}}{4}\left(2-X+u_{0} T\right)\right] \exp \left[\frac{u_{0}}{D_{0}}-\frac{\frac{1}{D_{0}}}{4 T}\left(2-X+u_{0} T\right)^{2}\right] \\
& -\frac{u_{0}}{D_{0}}\left[\left(2-X+\frac{3 u_{0} T}{2}\right)+\frac{\frac{u_{0}}{D_{0}}}{4}\left(2-X+u_{0} T\right)^{2}\right] \\
& \exp \left(\frac{u_{0}}{D_{0}}\right) \operatorname{erfc}\left[\frac{\frac{1}{D_{0}}(2-X)+\frac{u_{0} T}{D_{0}}}{2 \sqrt{\frac{T}{D_{0}}}}\right]
\end{aligned}
$$


2.2 Non-Uniform flow for space dependent dispersion coefficient

Now, the inhomogeneous porous domain is considered. In this case the flow is non-uniform and the values of $u$ and $D$ are taken as in [25]

$$
u(x, t)=u_{0}(1+a x), \quad D(x, t)=D_{0}(1+a x)^{2} .
$$

The Eq.(1) is solved for the velocity and dispersion coefficient given in Eq. (35) along with boundary conditions (3) and (6). As previously done in case of uniform flow, a new space variable $X$ is introduced through the following transformation.

$$
X=-\int \frac{d x}{(1+a x)^{2}}=\frac{1}{a(1+a x)} .
$$

The resulting PDE is further reduced by using a transformation with constant coefficient as follow:

$$
Z=-\log a X=\log (1+a x) .
$$

Using the transformations in Eqs. (36) and (37), we obtain the following reduced form of Eq. (1):

$$
\begin{gathered}
\frac{\partial C}{\partial t}=a_{1}^{2} D_{0} \frac{\partial^{2} C}{\partial Z^{2}}-\left(a_{1} u_{0}-a_{1}^{2} D_{0}\right) \frac{\partial C}{\partial Z}-a_{1} u_{0} C, \\
C(Z, 0)=0, \quad 0 \leq Z \leq Z_{0}, \quad Z_{0}=\log \left(1+a_{1} L\right) .
\end{gathered}
$$

Moreover, the transformed boundary conditions in terms of $Z$ and $t$ are given as following.

Type I boundary condition: The inlet boundary condition is given as

$$
C(Z, t)=\left\{\begin{array}{c}
C_{0}, \quad 0 \leq t \leq t_{i n j} \\
0, \quad t>t_{i n j}
\end{array}\right.
$$

The outlet Neumann boundary condition:

$$
\frac{\partial C(Z, t)}{\partial Z}=0, \quad Z=\infty, \quad t \geq 0 .
$$

Type II boundary condition:

The inlet and outlet boundary conditions are given as

$$
\begin{gathered}
-\frac{a_{1} D_{0}}{u_{0}-a_{1} D_{0}} \frac{\partial C}{\partial Z}+\left.C\right|_{Z=0}=\left\{\begin{array}{lr}
C_{0}, & 0<t \leq t_{i n j}, \\
0, & t>t_{i n j},
\end{array}\right. \\
\frac{\partial C(Z, t)}{\partial Z}=0, \quad Z=Z_{0}, \quad t \geq 0 .
\end{gathered}
$$

For the analytical solution of Eq. (38), we apply Laplace transformation to get

$$
\frac{\partial^{2} \bar{C}}{\partial Z^{2}}-\frac{a_{1} u_{0}-a_{1}^{2} D_{0}}{a_{1}^{2} D_{0}} \frac{\partial \bar{C}}{\partial Z}-\frac{\bar{C}\left(s+a_{1} u_{0}\right)}{a_{1}^{2} D_{0}}=0 .
$$

Therefore, the solution in Laplace domain is given as 


$$
\bar{C}(Z, s)=A e^{m_{1} Z}+B e^{m_{2} Z}
$$

where

$$
m_{1,2}=\frac{\left(a_{1} u_{0}-a_{1}^{2} D_{0}\right)}{2 a_{1}^{2} D_{0}} \pm \sqrt{\left(\frac{a_{1} u_{0}-a_{1}^{2} D_{0}}{2 a_{1}^{2} D_{0}}\right)^{2}+\frac{s+a_{1} u_{0}}{a_{1}^{2} D_{0}}}
$$

To determine the constants $A$ and $B$ in the above solution, the type I and type II boundary conditions are used in the following.

The Eqs. (40) and (41) are transformed in Laplace domain as given below

$$
\begin{gathered}
\bar{C}(Z, s)=\frac{C_{0}\left(1-e^{-s t_{i n j}}\right)}{s} \quad, Z=0, \\
\frac{\partial \bar{C}(Z, s)}{\partial Z}=0, \quad Z=\infty, \quad t \geq 0 .
\end{gathered}
$$

Using Eqs. (47) and (48) in Eq. (45), we get A and B as

$$
A=0
$$

$$
B=\frac{C_{0}\left(1-e^{-s t_{i n j}}\right)}{s}, Z=0 .
$$

Hence, the solution in Eq. (45) takes the form

$$
\bar{C}(Z, s)=\frac{C_{0}\left(1-e^{-s t_{i n j}}\right)}{s} e^{m_{2} Z} .
$$

As per previous methodology, the Laplace transformation is applied on type II boundary conditions given in Eqs. (42) and (43) to get

$$
\begin{gathered}
\bar{C}(Z, s)=\frac{C_{0}\left(1-e^{-s t_{i n j}}\right)}{s}+\frac{a D_{0}}{u_{0}-a D_{0}} \frac{\partial \bar{C}}{\partial Z}, \quad Z=0 . \\
\frac{\partial \bar{C}(Z, s)}{\partial Z}=0, \quad Z=Z_{0}, \quad t \geq 0 .
\end{gathered}
$$

So, using Eqs. (52) and (53), we get $A$ and $B$ as

$$
\begin{aligned}
& A=-\frac{C_{0}}{s}\left(1-e^{-s t_{i n j}}\right)\left[\frac{m_{2} e^{m_{2} Z_{0}}}{m_{1} e^{m_{1} Z_{0}}\left(1-\xi m_{2}\right)-m_{2} e^{m_{2} Z_{0}}\left(1-\xi m_{1}\right)}\right] . \\
& B=\frac{C_{0}}{s}\left(1-e^{-s t_{i n j}}\right)\left[\frac{m_{1} e^{m_{1} Z_{0}}}{m_{1} e^{m_{1} Z_{0}}\left(1-\xi m_{2}\right)-m_{2} e^{m_{2} Z_{0}}\left(1-\xi m_{1}\right)}\right] .
\end{aligned}
$$


Putting values of A and B in Eq. (45) to get the solution corresponding to Danckwerts boundary conditions as

$$
\begin{gathered}
\bar{C}(Z, s)=\frac{C_{0}\left(1-e^{-s t_{i n j}}\right)}{s\left(m_{1} e^{m_{1} Z_{0}}\left(1-\xi m_{2}\right)-m_{2} e^{m_{2} Z_{0}}\left(1-\xi m_{1}\right)\right)} \\
{\left[m_{1} e^{m_{1} Z_{0}+m_{2} Z}-m_{2} e^{m_{2} Z_{0}+m_{1} Z}\right],}
\end{gathered}
$$

where

$$
\xi=\frac{a_{1} D_{0}}{u_{0}-a_{1} D_{0}}
$$

In this case numerical Laplace inversion is applied to get back the solution in actual time domain.

\section{Moment analysis}

In this section, method of moments is used to figure out important information related to mass transfer kinetics and retention time in the given domain [32,30]. The Laplace transformation is used as a fundamental tool to get the moments. The optimum solution of the equation is provided by numerical Laplace inversion, but to investigate the behavior of elution profiles this solution does not help. The parameters of the mass transfer kinetics and the retention equilibrium-constant in the column are relevant to the moments in the Laplace domain. Here, a method for describing peaks by means of statistical moments is used.

The $n$-th temporal moment of column of length $x=L$ at inlet is

$$
\mu_{n}=\int_{0}^{\infty} C(t, x=L) t^{n} d t
$$

The normalized temporal moment is defined as

$$
\mu_{n}=\frac{\int_{0}^{\infty} C(t, x=L) t^{n} d t}{\int_{0}^{\infty} C(t, x=L) d t} .
$$

While, the $n$-th central moment is

$$
\mu_{n}^{\prime}=\frac{\int_{0}^{\infty} C(t, x=L)\left(t-\mu_{1}\right)^{n} d t}{\int_{0}^{\infty} C(t, x=L) d t} .
$$

In this section, the first three moments are derived for all sets of considered BCs. The zeroth moment $\mu_{0}$ represents total mass of the solute. The first moment $\mu_{1}$ corresponds to the retention time. The second central moment $\mu_{2}^{\prime}$ or the variance of the elution breakthrough curves (or peaks) provides significant information related to processes of mass transfer in the domain. The third central moment $\mu_{3}^{\prime}$ is considered to analyze the fronts asymmetries. The second and the third central moment for the more general Danckwerts BCs reduce to the moments for the Dirichlet BCs in case $D_{0}$ approaches to zero. A comparison of analytical moments and numerical moments is made. 
3.1 Moments of Uniform flow for time dependent dispersion coefficient

Case I: Dirichlet boundary conditions

Let us define

$$
n_{1}=\frac{u_{0}}{D_{0}}
$$

The zeroth moment:

$$
\mu_{0}=C_{0} T_{\mathrm{inj}}
$$

The first moment:

$$
\mu_{1}=\frac{T_{\mathrm{inj}}}{2}+\frac{X_{0}}{u_{0}}
$$

The second central moment:

$$
\mu_{2}^{\prime}=\frac{T_{\mathrm{inj}}^{2}}{12}+\frac{2 X_{0}}{n_{1} u_{0}^{2}}
$$

Third central moment:

$$
\mu_{3}^{\prime}=12 \frac{X_{0} D^{2}}{n_{1}^{2} u 0^{3}} .
$$

This completes the moments obtained for type I boundary condition.

\section{Case II: Danckwerts boundary conditions}

The zeroth moment is obtained as

$$
\mu_{0}=C_{0} T_{\mathrm{inj}}
$$

First moment is calculated as

$$
\mu_{1}=\frac{T_{\mathrm{inj}}}{2}+\frac{X_{0}}{u_{0}}
$$

The second moment is

$$
\mu_{2}=\frac{T_{\mathrm{inj}}^{2}}{3}+T_{\mathrm{inj}} \frac{X_{0}}{u_{0}}+\frac{2}{n_{1}^{2} u_{0}^{2}}\left[-1+X_{0} n_{1}+\frac{X_{0}^{2} p_{1}^{2}}{2}+e^{-X_{0} n_{1}}\right] .
$$

The second central moment is

$$
\mu_{2}^{\prime}=\frac{T_{\mathrm{inj}}^{2}}{12}+\frac{2 X_{0}}{n_{1} u_{0}^{2}}\left[1+\frac{1}{X_{0} n_{1}}\left(e^{-X_{0} n_{1}}-1\right)\right] .
$$

Finally, the third central moment is

$$
\mu_{3}^{\prime}=\frac{12 X_{0}}{n_{1}^{2} u_{0}^{3}}\left[\left(1+\frac{2}{X_{0} n_{1}}\right) e^{-X_{0} n_{1}}+\left(1-\frac{2}{X_{0} n_{1}}\right)\right] .
$$


3.2 Moments of Non-uniform flow for space dependent dispersion coefficient

Case I: Dirichlet boundary conditions

We calculate moments for the solution of infinity Neumann boundary condition.

The zeroth moment:

$$
\mu_{0}=\left(C_{0} t_{\text {inj }}\right) e^{\frac{1}{2}\left(\alpha_{2}\right) Z_{0}}
$$

The first moment:

$$
\mu_{1}=\frac{t_{\text {inj }}}{2 C_{0}}+\frac{p_{3} Z_{0}}{\sqrt{\alpha_{1}}}
$$

The second central moment:

$$
\begin{aligned}
\mu_{2}^{\prime}= & \frac{1}{12 C_{0}^{2}\left(\alpha_{1}\right)^{\frac{3}{2}}}\left[\sqrt{\alpha_{1}}\left(16 C_{0}^{2} p_{2}+4 C_{0}^{2} p_{1}^{2}-3 p_{1}^{2}-12 p_{2}\right) t_{\mathrm{inj}}^{2}\right. \\
& \left.+12 C_{0} p_{3} Z_{0}\left(4 p_{2} C_{0}+C_{0} p_{1}^{2}-p_{1}^{2}-4 p_{2}\right) t_{\mathrm{inj}}+24 p_{3}^{2} Z_{0} C_{0}^{2}\right] .
\end{aligned}
$$

Third central moment:

$$
\begin{aligned}
\mu_{3}^{\prime}= & -\frac{1}{4 C_{0}^{3}\left(\alpha_{1}\right)^{\frac{5}{2}}}\left[\sqrt { \alpha _ { 1 } } \left(8 C_{0}^{3} p_{1}^{2} p_{2}+16 C_{0}^{2} p_{1}^{2} p_{2}-p_{1}^{4}\right.\right. \\
& \left.-16 p_{2}^{2}-16 C_{0}^{3} p_{2}^{2}-C_{0}^{3} p_{1}^{4}-8 p_{1}^{2} p_{2}+32 C_{0}^{2} p_{2}^{2}+2 C_{0}^{2} p_{1}^{4}\right) t_{\mathrm{inj}}^{3} \\
& +C_{0} p_{3} Z_{0}\left(48 p_{2} C_{0} p_{1}^{2}-48 p_{1}^{2} p_{2}+96 C_{0} p_{2}^{2}+6 C_{0} p_{1}^{4}-6 p_{1}^{4}-96 p_{2}^{2}\right) t_{\mathrm{inj}}^{2} \\
& +C_{0}^{2} p_{3}^{2} Z 0\left(-6 \sqrt{\alpha_{1}} Z_{0} p_{1}^{2}-24 Z_{0} p_{2} \sqrt{\alpha_{1}}+24 C_{0} p_{2} \sqrt{\alpha_{1}}+6 C_{0} Z_{0} \sqrt{\alpha_{1}} p_{1}^{2}\right. \\
& \left.\left.-48 C_{0} p_{2}-12 C_{0} p_{1}^{2}+12 p_{1}^{2}+48 p_{2}\right) t_{\mathrm{inj}}-48 C_{0}^{3} p_{3}^{3} Z 0\right] .
\end{aligned}
$$

\section{Case II: Danckwerts boundary conditions}

For the moments in the case of non-uniform Danckwerts boundary condition. The zeroth moment:

$$
\mu_{0}=-\frac{2 C_{0} t_{\mathrm{inj}} p_{1} \sqrt{\alpha_{1}} e^{p_{1} Z_{0}}}{\alpha_{8} e^{\frac{1}{2} \alpha_{2} Z_{0}}-\alpha_{9} e^{\frac{1}{2} \alpha_{3} Z_{0}}} .
$$

The first moment:

$$
\begin{gathered}
\mu_{1}=\frac{1}{2 \alpha_{1}\left(\alpha_{8} e^{\frac{1}{2} \alpha_{2} Z_{0}}-\alpha_{9} e^{\frac{1}{2} \alpha_{3} Z_{0}}\right)}\left[\left(\alpha_{10} e^{\frac{1}{2} \alpha_{2} Z_{0}}-\alpha_{11} e^{\frac{1}{2} \alpha_{3} Z_{0}}\right) t_{\mathrm{inj}}+\right. \\
\left.\left(\alpha_{12} e^{\frac{1}{2} \alpha_{2} Z_{0}}-\alpha_{13} e^{\frac{1}{2} \alpha_{3} Z_{0}}\right)\right] .
\end{gathered}
$$

Here to simplify the calculations some terms like $p_{1}, p_{2}, p_{3}, z_{0}, \alpha_{1}, \alpha_{2}, \alpha_{4}, \alpha_{5}, \alpha_{6}$, $\alpha_{7}, \alpha_{8}, \alpha_{9}, \alpha_{10}, \alpha_{11}, \alpha_{12}, \alpha_{13}, \alpha_{14}, \alpha_{15}$ have been defined and given in Appendix A.

Moreover, the second moment and second central moment are too lengthy to write. Thus, we will use them in the simulation of the results. 


\section{Results and discussions}

In this section, the analytical solutions obtained for two set of boundary conditions are validated. For this purpose a high resolution finite volume scheme (HR-FVS) of Koren [34] is used. This scheme guarantees the positivity of the solution. Initially, first case is discussed, i.e., solute dispersion which is dependent on time in uniform flow. Later on second case is discussed, i.e., solute dispersion which is space dependent in non-uniform flow.

For time dependent solute dispersion the analytical and numerical results are compared considering Danckwerts BCs along uniform and non-uniform flows. Table 1 presents all the parameters used in the problem. The comparison of numerical and analytical results of the advection-diffusion equation along with Danckwerts BCs are displayed in Figures 1 and 2. A close agreement of the elution profiles verify the correctness of the analytical solutions and accuracy of the Koren scheme. In figures 1 and 2 it can be noticed that local concentration profile appeared at 2 minutes for uniform flow and for non-uniform flow it appeared before 2 minutes which means uniform flow takes more time to reach at domain outlet as compared to non-uniform flow. Also it can be seen that concentration profile is close to 1 for uniform flow whereas in non-uniform flow its concentration profile is close to 0.5. Importantly, it was observed that the numerical Laplace inversion technique is more appropriate for solving such problems and it is a consistent tool for solving problems especially when it is not possible to find out the exact solution. Figures 3 and 4 shows the comparison of Dirichlet and Danckwerts BCs for different values of dispersion coefficient $D_{0}$ along uniform flow and non-uniform flow respectively, where injection time is taken equal to $1 \mathrm{~min}$. From these figures, it can be observed that when the value of $D_{0}$ is taken very small i.e. 0.002 , there is no variation in the solutions of Dirichlet and Danckwerts BCs, whereas, for larger values of $D_{0}$ a visible difference between the results obtained from both set of BCs can be seen. Moreover, influence of different time injection for the uniform flow case is shown in Figure 5.

\section{Description on the numerically and analytically determined moments}

This section focuses on numerically and analytically determined temporal moments with considered BCs. Numerical moments are obtained with the help of formulas in Eqs. (59) and (60) for the first normalized moment, second central moment and third central moment. The integral appearing in these equations are numerically approximated by using trapezoidal rule. For the moment calculations of rectangular pulse, the injection time is taken to be $t_{\mathrm{inj}}=3 \mathrm{~min}$. The Danckwerts BCs were found to be more accurate than Dirichlet BCs thus Danckwerts BCs are taken into account for test problems.

A comparison of analytical and numerical moments for both uniform and nonuniform flows are shown in the Figures 6 and 7 . The zeroth moment $\mu_{0}:$ denotes the total mass injected to the column, the first moment $\mu_{1}$ : represents the retention time of the pulse in the column, the second central moment $\mu_{2}^{\prime}$ : corresponds to the variance of the profile and the third central moment $\mu_{3}^{\prime}$ : describes the skewness (asymmetry) of the concentration profile. Both numerical and analytical 
moments agree well with each other that verifies the accuracy of proposed numerical scheme and correctness of the analytical solutions. These plots show that retention time, variance and asymmetry of the profile are reducing on increasing the intersticial velocity $u$. In Figure 7 the visible difference (discrepancy) between analytical and numerical $\mu_{2}$ for small value of velocity $u_{0}$ (or for large $1 / u_{0}^{2}$ ) is due to numerical diffusion and errors introduced by small time steps and large number of iterations. Thus, the numerical solution has actually more errors due to the combined effects of numerical diffusion and axial dispersion.

Table 1 Parameters of the model under consideration

\begin{tabular}{|c|c|}
\hline Parameters & values \\
\hline Column length & $L=1.0 \mathrm{~cm}$ \\
Porosity & $\epsilon=0.4$ \\
Interstitial velocity & $u_{0}=1.0 \mathrm{~cm} / \mathrm{min}$ \\
Dispersion coefficient & $D_{0}=0.002 \mathrm{~cm}^{2} / \mathrm{min}$ \\
Injection time for uniform flow & $T_{\mathrm{inj}}=3.0 \mathrm{~min}$ \\
Injection time for non-uniform flow & $t_{\text {inj }}=3.0 \mathrm{~min}$ \\
Concentration at inlet & $C_{0}=1.0 \mathrm{~g} / \mathrm{l}$ \\
Adsorption equilibrium constant & $a=1.0$ \\
Parameter accounting in-homogeneity & $a_{1}=1.0$ \\
\hline
\end{tabular}




\section{Conclusion}

In this work, one-dimensional advection-diffusion model with variable coefficients was analyzed analytically and numerically. Two case studies along with two different boundary conditions, namely, the Dirichlet and Danckwerts boundary conditions were considered. In the first case temporal dependent solute dispersion was studies in homogeneous domain for uniform flow. While, in the second case space dependent velocity was considered and the dispersion was assumed proportional to the square of the velocity for non-uniform flow. Laplace transformation was used as basic tool to find the analytical solutions. However, in the cases where the analytical inversion was not possible, numerical Laplace inversion was used to obtain the concentration profiles in actual time domain. Temporal moments upto third order were calculated from analytical solution in Laplace domain. Analytical results were validated against numerical result by using HR-FVS. A good agreement between analytical and numerical results reveals that the HR-FVS can be used to solve such advection-diffusion type model equations. 


\section{Appendix A}

Let us define:

$$
\begin{aligned}
& p_{1}=\frac{a_{1} u_{0}-a_{1}^{2} D_{0}}{a_{1}^{2} D_{0}}, \quad p_{2}=\frac{u_{0}}{a_{1} D_{0}}, \\
& p_{3}=\frac{1}{a_{1}^{2} D_{0}}, \quad Z_{0}=\log \left(1+a_{1} X_{0}\right), \\
& \alpha_{1}=p_{1}^{2}+4 p_{2}, \quad \alpha_{2}=p_{1}-\sqrt{\alpha 1}, \quad \alpha_{3}=p_{1}+\sqrt{\alpha 1} \\
& \alpha_{4}=4 p_{2} p_{1}+p_{1}^{3}-\alpha_{1}^{\frac{3}{2}}, \quad \alpha_{5}=4 p_{2} p_{1}+p_{1}^{3}+\alpha_{1}^{\frac{3}{2}}, \\
& \alpha_{6}=8 p_{3} Z_{0} p_{2}+2 p_{3} Z_{0} p_{1}^{2}-4 p_{3} p_{1}-2 \sqrt{\alpha_{1}} p_{3} Z_{0} p_{1} \\
& \alpha_{7}=8 p_{3} Z_{0} p_{2}+2 p_{3} Z_{0} p_{1}^{2}-4 p_{3} p_{1}+2 \sqrt{\alpha_{1}} p_{3} Z_{0} p_{1} \\
& \alpha_{8}=p_{1}^{2}-p_{1} \sqrt{\alpha_{1}}+2 p_{2}, \quad \alpha_{9}=p_{1}^{2}+p_{1} \sqrt{\alpha_{1}}+2 p_{2}, \\
& \alpha_{10}=p_{1}^{4}-p_{1} \alpha_{1}^{\frac{3}{2}}+8 p_{2}+6 p_{1}^{2} p_{2}, \quad \alpha_{11}=p_{1}^{4}+p_{1} \alpha_{1}{ }^{\frac{3}{2}}+8 p_{2}+6 p_{1}^{2} p_{2}, \\
& \alpha_{12}=2 p_{3} Z_{0} p_{1}^{3}+8 p_{3} p_{2}+8 p_{3} Z_{0} p_{2} p_{1}-\left(\sqrt{\alpha_{1}} p_{3} Z_{0} p_{1}^{2}+p_{3} Z_{0} \alpha_{1}^{\frac{3}{2}}\right) \\
& \alpha_{13}=2 p_{3} Z_{0} p_{1}^{3}+8 p_{3} p_{2}+8 p_{3} Z_{0} p_{2} p_{1}+\left(\sqrt{\alpha_{1}} p_{3} Z_{0} p_{1}^{2}+p_{3} Z_{0} \alpha_{1}^{\frac{3}{2}}\right), \\
& \alpha_{14}=2816 p_{2}^{4} p_{1}^{2}+784 p_{2}^{2} p_{1}^{2}+8 p_{10}+2240 p_{2}^{3} p_{1}^{4}+1024 p_{2}^{5}+128 p_{2} p_{1}^{8} \\
& -p_{1}^{3}\left(4 p_{1}^{5} \alpha_{1}^{\frac{5}{2}}+16 p_{2} p_{1} \alpha_{1}^{\frac{7}{2}}+16 p_{2} \alpha_{1}^{\frac{5}{2}}+4 \alpha_{1}^{\frac{7}{2}}\right) \text {, } \\
& \alpha_{15}=2816 p_{2}^{4} p_{1}^{2}+784 p_{2}^{2} p_{1}^{2}+8 p_{10}+2240 p_{2}^{3} p_{1}^{4}+1024 p_{2}^{5}+128 p_{2} p_{1}^{8} \\
& +p_{1}^{3}\left(4 p_{1}^{5} \alpha_{1}^{\frac{5}{2}}+16 p_{2} p_{1} \alpha_{1}^{\frac{7}{2}}+16 p_{2} \alpha_{1}^{\frac{5}{2}}+4 \alpha_{1}^{\frac{7}{2}}\right) \text {. }
\end{aligned}
$$




\section{References}

1. Lanser, D., and Jan G. Verwer.: Analysis of operator splitting for advection-diffusionreaction problems from air pollution modelling. Journal of computational and applied mathematics 111 (1) 201-216(1999)

2. Samadi-Dooki, Aref, Hossein M. Shodja, and Leila Malekmotiei.: The effect of the physical properties of the substrate on the kinetics of cell adhesion and crawling studied by an axisymmetric diffusion-energy balance coupled model. Soft matter 11 (18) 3693-3705(2015)

3. Grisak, G. E., and J. F. Pickens.: An analytical solution for solute transport through fractured media with matrix diffusion. Journal of Hydrology 52 (1-2) 47-57(1981)

4. Schwarz, Harold A.: Applications of the spur diffusion model to the radiation chemistry of aqueous solutions. The Journal of Physical Chemistry 73 (6) 1928-1937(1969)

5. Sameena B., Qamar, S., Seidel-Morgenstern, A.: Irreversible and reversible reactive chromatography: Analytical solutions and moment analysis for rectangular pulse injections. Journal of Chromatography A, 1385 49-62(2015)

6. Ogata, A. and Banks, R. B.: A solution of the differential equation of longitudinal dispersion in porous media. US Geol. Surv. Prof. Paper, 411-A 1-9(1961)

7. Harleman, D. R. F., and Rumer, R. R.: Longitudinal and lateral dispersion in an isotropic porous medium. J. Fluid Mech., 385-394(1963)

8. Bear, J.: Dynamics of fluids in porous media (New York: Amr. Elsev. Co.), (1972)

9. Guvanasen, V., and Volker, R. E.: Experimental investigations of unconfined aquifer pollution from recharge basins. Water Resour. Res., 19(3) 707-717(1983).

10. Aral, M. M., and Liao, B.: Analytical solutions for two-dimensional transport equation with time-dependent dispersion coefficients. J. Hydrol. Engg., 1(1) 20-32(1996)

11. Jensen, J.L. and Lake, L.W.: The influence of sample size and permeability distribution on heterogeneity measures. SPE Reservoir Engineering, 3(2) 629-637(1988)

12. Perkins, T.K. and Johnston, O.C.: A Review of Diffusion and Dispersion in Porous Media. SPE Reprint Series, No 8 or Soc. Pet. Eng. Jour., 3 70(1963)

13. Banks, R. B., and Ali, J.: Dispersion and adsorption in porous media flow. J. Hydraul. Div., 90 13-31(1964)

14. Al-Niami, A. N. S., and Rushton, K. R. Analysis of flow against dispersion in porous media. J. Hydrol., 33 87-97(1977)

15. Thongmoon M., McKibbin, R.: A Comparison of Some Numerical Methods for the Advection-Diffusion Equation. Res. Lett. Inf. Math. Sci., 10 49-62(2006)

16. Kitahara, N., Nagahara, D., Yano, H. A Numerical Inversion of Laplace Transform and its Application. Journal of the Franklin Institute, 325 (2) 221-233 (1988)

17. Hassanzadeh, Hassan and Pooladi-Darvish Mehran. Comparison of different numerical Laplace inversion methods for engineering applications. Applied Mathematics and Computation, 189 (2) 1966-1981(2007)

18. Stehfest, H. Numerical inversion of Laplace transforms algorithm. Comm. ACM, 13 (1) 47-49 (1979)

19. Bellman, R., Kalaba, R. E., and Lockett, J. A.: Numerical Inversion of the Laplace Transform, Elsevier, New York (1966)

20. Zakian, Littlewood, R. K.: Numerical inversion of Laplace transforms by weighted leastsquares approximation, Comput. J., 16(1) 66-68(1972)

21. Wilcox, D. J., Gibson, I. S.: Numerical transformation and inversion in the analysis of physical systems. Int. J. Num. Methods Eq., 20 1507- 1519(1984)

22. Hosono, T.: Numerical inversion of Laplace transform. J. Inst. Elect. Comm. Eng. Japan, 15(8) 849-854(1982)

23. Veillon, F.: Numerical inversion of Laplace transform. Collected Algorithms from CACM, Algorithm, 486 587-589(1972)

24. Duffy, D. G.: Transform Methods for Solving Partial Differential Equations, Second Edition, Chapman and Hall/CRC,(2004)

25. Kumar, A., Jaiswal, D. K., and Kumar, N.: Analytical Solutions of One-Dimensional Advection-Diffusion Equation with Variable Coefficients in a Finite Domain, Journal of Earth System Science, 118 539-549 (2009)

26. Jaiswal, D. K., Kumar, A., Yadav, R. R.: Analytical Solution to the One-Dimensional Advection-Diffusion Equation with Temporally Dependent Coefficients, Journal of Water Resource and Protection, 3 76-84 (2011)

27. Van, Kooten, J. J. A.: A method to solve the advection-dispersion equation with a kinetic adsorption isotherm. Adv. Water Res., 19 193-206(1996) 
28. Zoppou, C., and Knight, J. H.: Analytical solutions for advection and advection-diffusion equation with spatially variable coefficients. J. Hydraul. Engg., 123 144-148(1997)

29. Felinger, A., Guiochon, G.: Comparison of the Kinetic Models of Linear Chromatography. Chromatographia Supplement 60, S175-S180(2004)

30. Guiochon G., Felinger A., Shirazi D. G., Katti A. M.: Fundamentals of Preparative and Nonlinear Chromatography, second ed., Elsevier Academic press, New York, (2006)

31. Kirkland, J. J., Truszkowski, F. A., Dilks, C. H., Engel, G.S.: Superficially porous silica microspheres for fast high-performance liquid chromatography of macromolecules. J. Chromatography A, 890 3-13(2000)

32. Qamar, S., Seidel-Morgenstern, A.: Extending the potential of moment analysis in chromatography, Trends in Analytical Chemistry 81, 87101(2016)

33. Gupta, A.D, Lake, L.W., Pope, G.A., Sepehrnori, K., and King, M.J.: High resolution monotonic schemes for reservoir fluid flow simulation. IN SITU, 15 (3), 289-317(1991)

34. Javeed, S., Qamar, S., Seidel-Morgenstern, A., Warnecke, G.: Efficient and accurate numerical simulation of nonlinear chromatographic processes. Computers and Chemical Engineering, 35 2294-2305(2011)

35. Van Genuchten, M. Th.: Analytical solutions for chemical transport with simultaneous adsorption, zeroth-order prodution and first order decay. J. Hydrol., 49 213-233 (1981) 


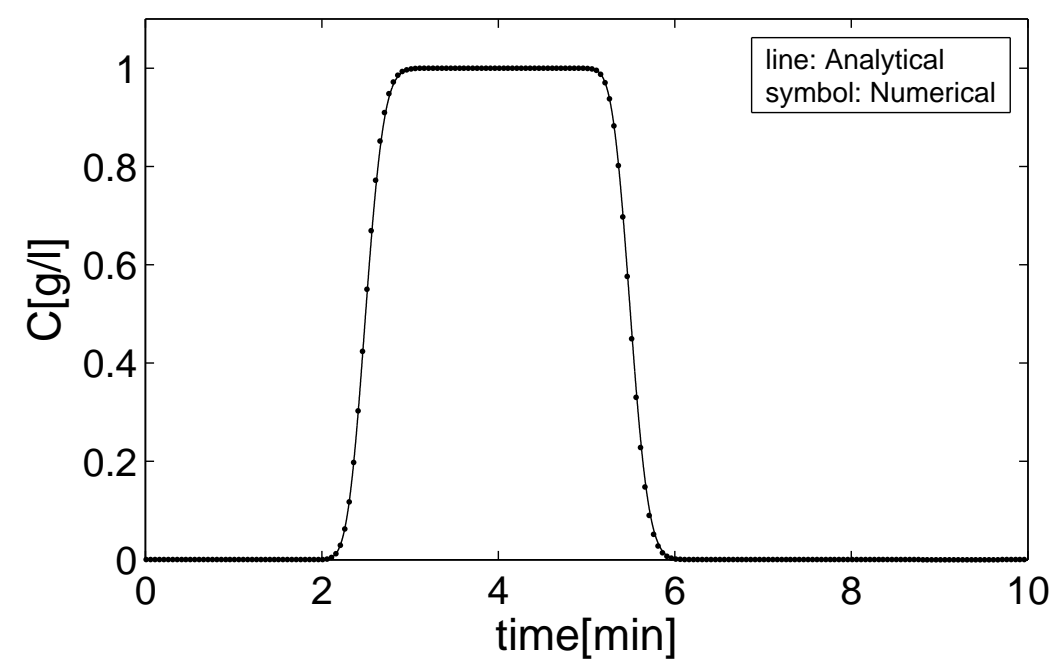

Fig. 1 Comparison of analytical and numerical results with Danckwerts BCs for uniform flow. All parameters are given in Table 1

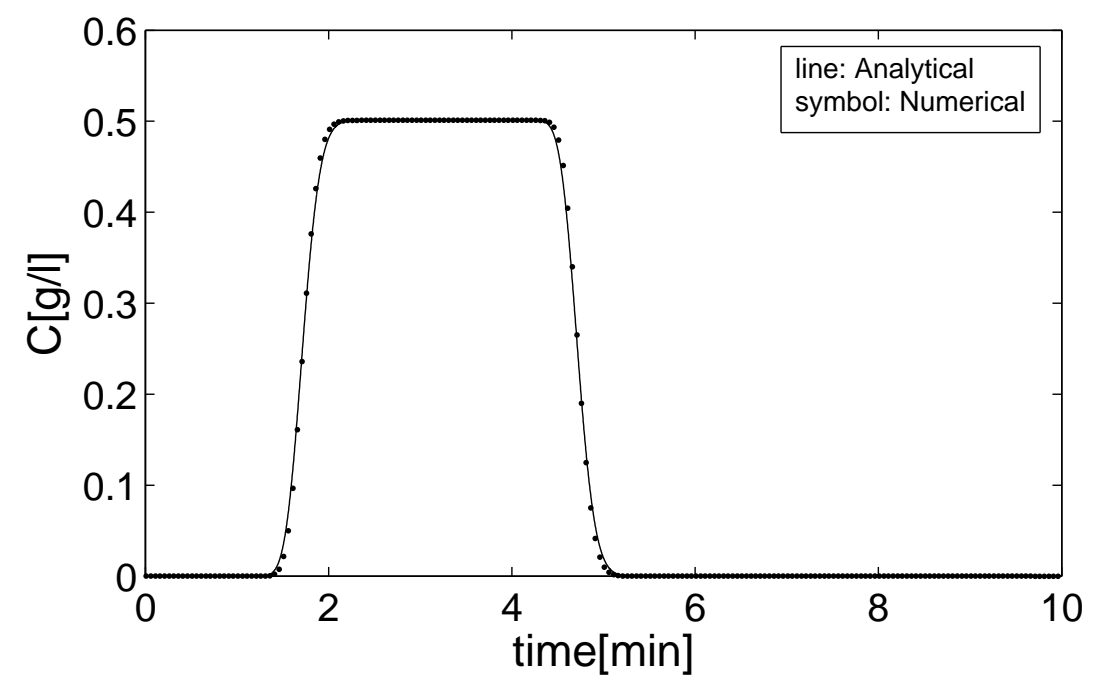

Fig. 2 Comparison of analytical and numerical results with Danckwerts BCs for non-uniform flow. 


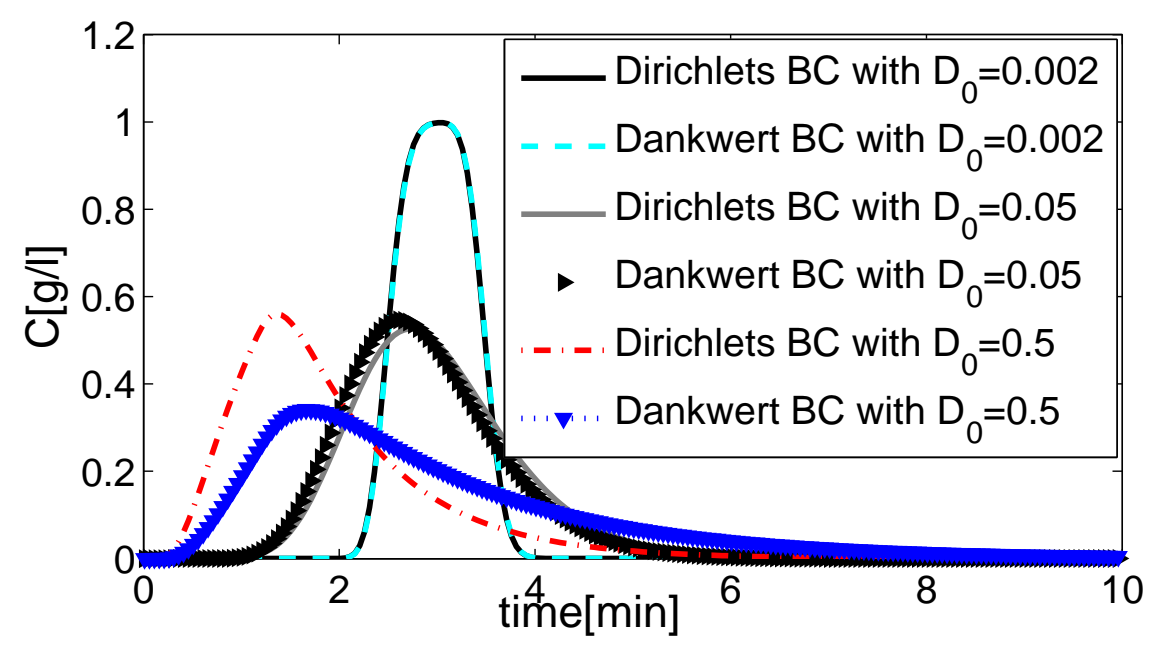

Fig. 3 Comparison of Dirichlet and Danckwerts BCs for different values of $D_{0}$ along uniform flow.

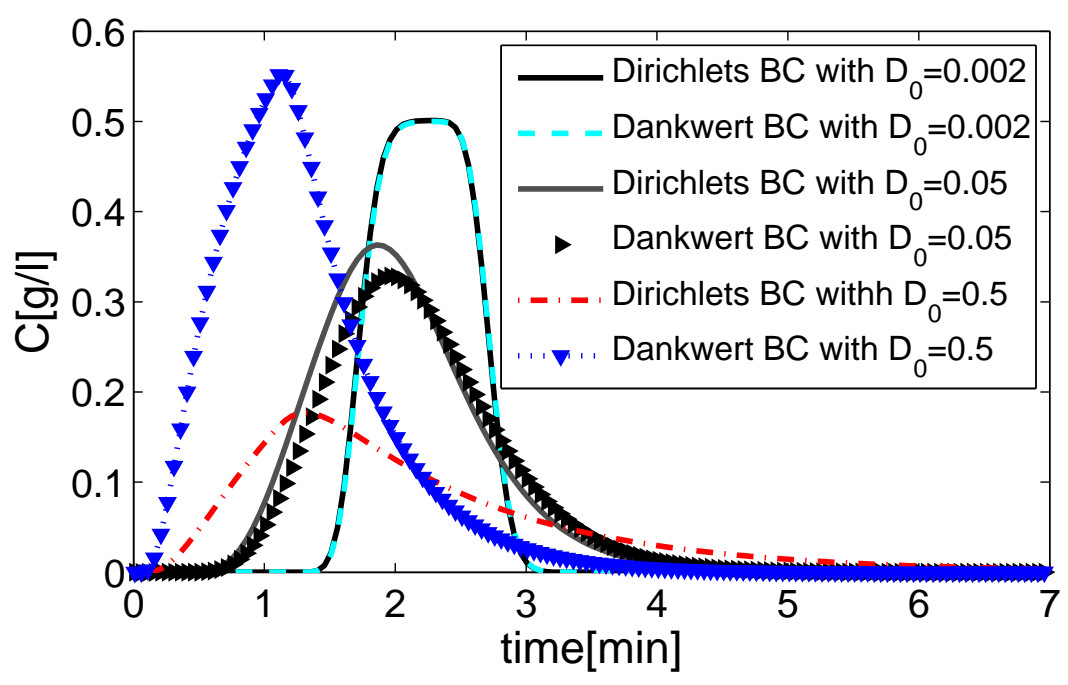

Fig. 4 Comparison of Dirichlet and Danckwerts BCs for different values of $D_{0}$ along nonuniform flow. 


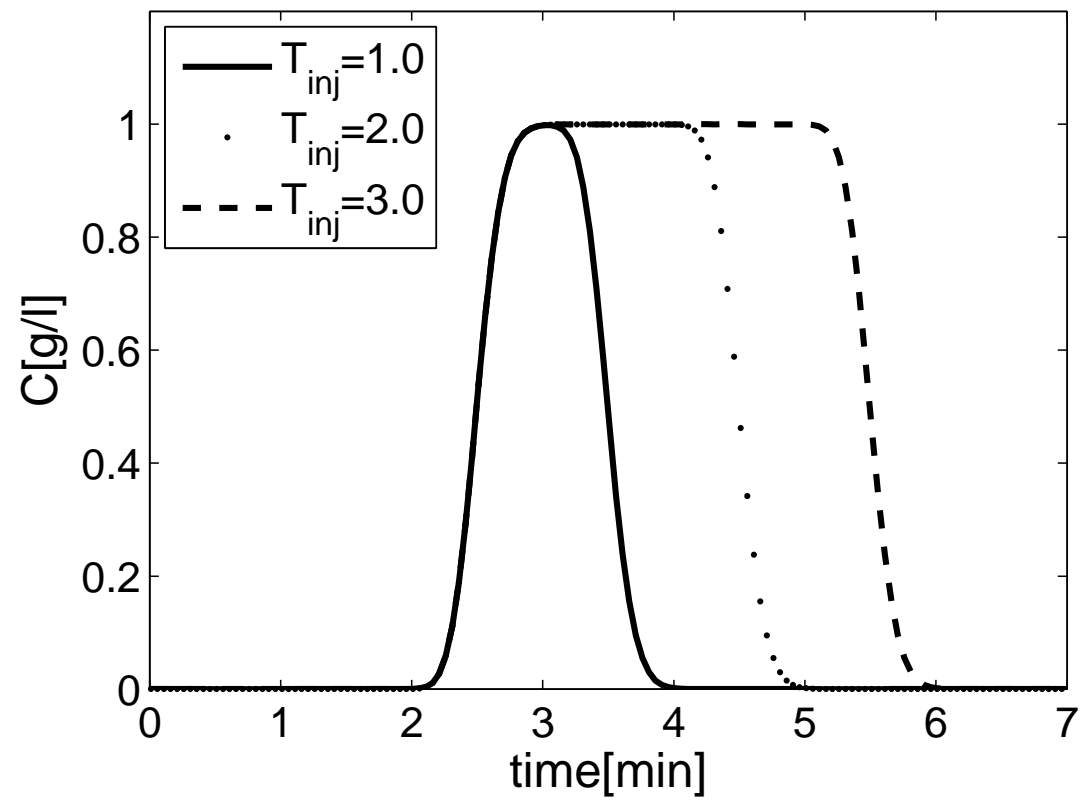

Fig. 5 Influence of different time injection using Danckwerts inlet BCs for uniform flow . 

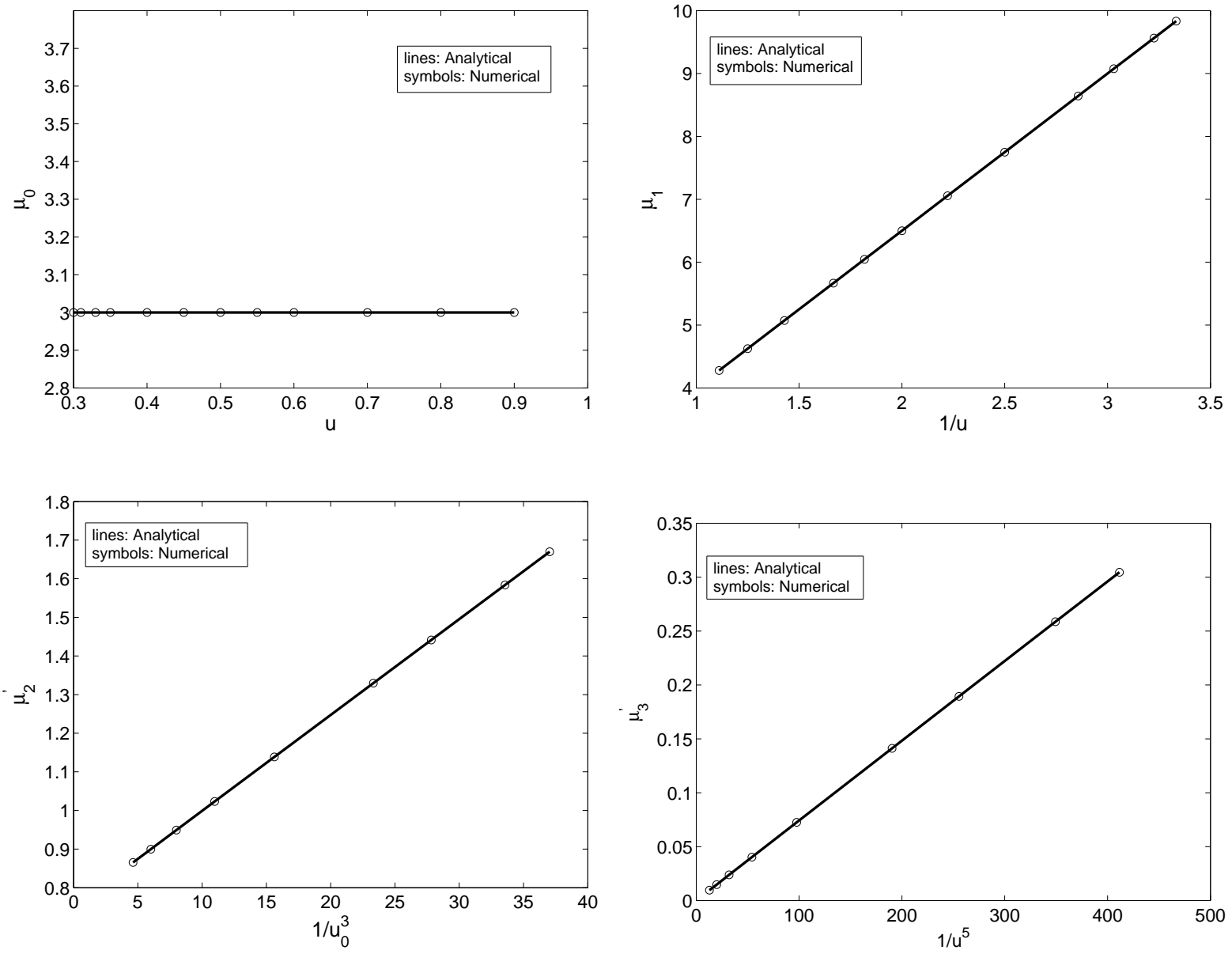

Fig. 6 Comparison of moments using Danckwerts BCs for uniform flow 

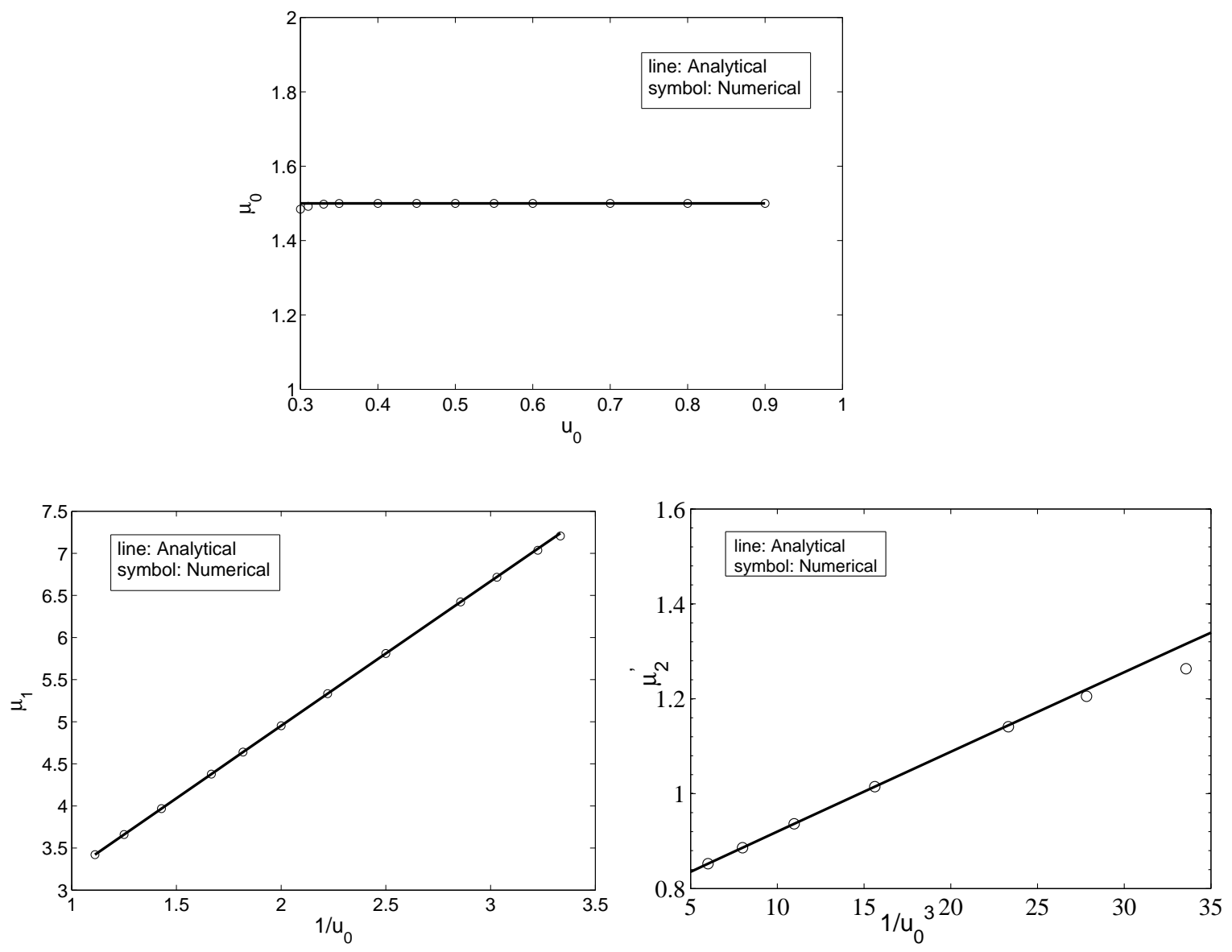

Fig. 7 Comparison of moments using Danckwerts BCs for non-uniform flow 